\title{
Drag Enhancement for Atmospheric Entry Capsule using Electrodynamic Effect with Multi-magnetic Source
}

\author{
By Yasunori NAGATA, ${ }^{1)}$ Kazuhiko YAMADA ${ }^{2)}$ and Takashi $\mathrm{ABE}^{2)}$ \\ ${ }^{1)}$ Graduate School of Natural Science and Technology, Okayama University, Okayama, Japan \\ ${ }^{\mathrm{m})}$ Institute of Space and Astronautical Science, JAXA, Sagamihara, Japan
}

(Received July 31st, 2015)

\begin{abstract}
In the electrodynamic flow control, weakly-ionized plasma flow behind the strong shock wave could be controlled by the applied magnetic field around a reentry vehicle. To control the flow field, a very strong magnetic field is required and it could be applied by the superconducting magnet, which is too large and heavy for a reentry capsule. Thus, in the present study, to avoid the use of the superconducting magnet, the electrodynamic effect from the combination of multiple weaker magnetic source such as permanent magnets is numerically investigated. According to the MHD simulation, the influence on the drag force caused by the multiple magnetic source, which is placed equiangularly around the body axis, could be diminished by the Hall effect. When the Hall effect is significant, the induced electric current intensity is very small because the electric field is generated much weaker than the one of the single magnet case. Therefore, the present magnetic configuration using multiple magnetic source might not be effective under the high Hall parameter condition such as the reentry flight.
\end{abstract}

Key Words: Hypersonic, Weakly Ionized Plasma, MHD

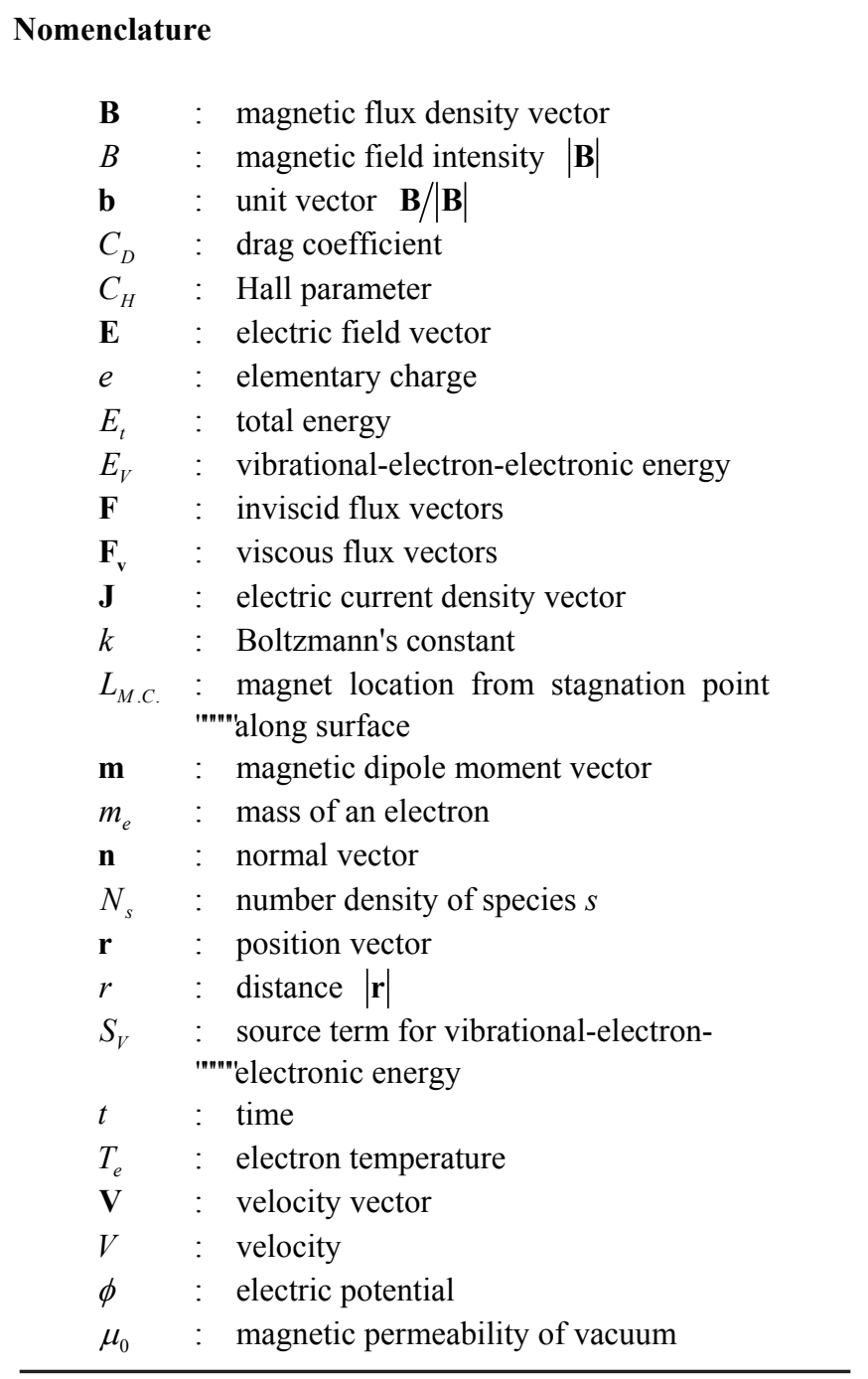

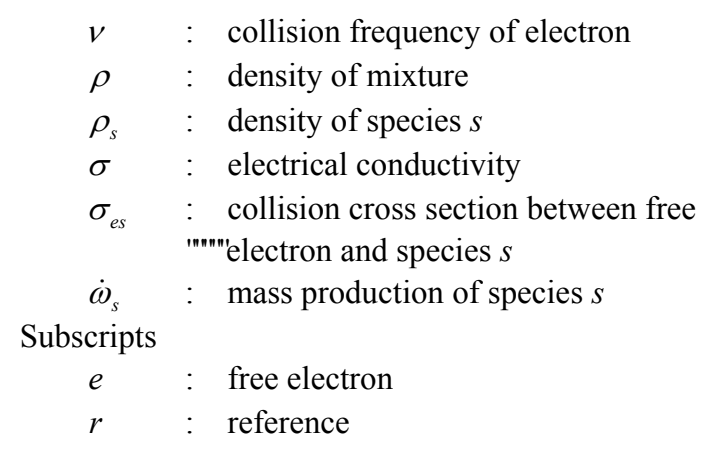

\section{Introduction}

The weakly ionized plasma flow around atmospheric entry vehicles could be controlled by utilizing applied magnetic field because of the Lorentz force acting on the plasma flow. This electrodynamic flow control has a potential to be a new type of the thermal protection system and it has been, both experimentally and numerically, investigated since 1950s. ${ }^{1-3)}$ Figure 1 shows the concept of the electrodynamic flow control generated in a hypersonic flow around a vehicle. A weakly ionized flow caused by the strong shock wave ahead of the vehicle interacts with an applied magnetic field $\mathbf{B}$ generated by the magnet inside the vehicle, and thus, the electric current $\mathbf{J}$ are induced in the plasma flow $\mathbf{V}$. Therefore, the Lorentz force $\mathbf{J} \times \mathbf{B}$ acts and it could alter the plasma flow; the shock layer and the drag force could be enhanced. We can expect a possible application of this effect to an active flow control without moving parts. ${ }^{4)}$

In the electrodynamic flow control, very strong magnet source, such as the superconducting magnet, should be required to obtain the clear advantage. However, the 


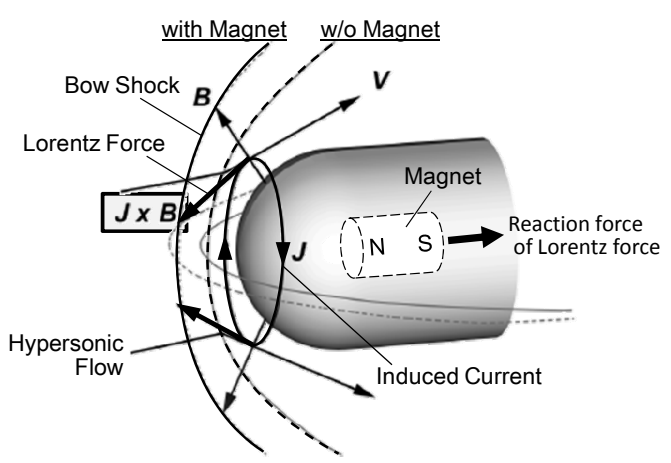

Fig. 1. Schematic view of the electrodynamic flow control.

superconducting magnet system is large and heavy for a reentry capsule such as the MUSES-C capsule, because it consists of a superconducting magnetic coil, an electric current source, a cooling mechanism, and refrigerant, etc. If the strong magnetic field source is installed, the majority payload space will be omitted from internal capsule space and it no longer works as the space transportation system. Then, the compact and light-weight system must be developed for the practical use.

To avoid the use of the superconducting magnet, the combination of the permanent magnets could be used for another approach to obtain the electrodynamic flow control effect. The permanent magnet, such as a neodymium magnet, could realize the compact and light-weight magnetic field source system because it requires no additional equipment if it is magnetized in advance. However, the magnetic field generated by the permanent magnet is generally weaker than the superconducting magnet. Then, to deal with this obstacle, we suggested the magnetic field source using many permanent magnets, which is named as the multiple magnetic source in this paper.

In the present study, the influence on the drag force caused by the multiple magnetic source is numerically investigated by means of the 3-dimensional magnetohydrodynamic (MHD) simulation. For this purpose, the MUSES-C capsule with the magnetic source is assumed and the several magnetic configuration cases generated by the multiple magnetic source are calculated under an atmospheric entry flight condition.

\section{Computational Methods}

\subsection{Governing equations}

The 3-dimensional Navier-Stokes equations with the electromagnetic effect are used to simulate the flow field around the atmospheric entry capsule with the multiple magnetic source, which are presented as,

$$
\frac{\partial}{\partial t}\left[\begin{array}{c}
\rho_{s} \\
\rho \mathbf{V} \\
E_{t} \\
E_{V}
\end{array}\right]+\nabla \cdot\left(\mathbf{F}-\mathbf{F}_{\mathbf{v}}\right)=\left[\begin{array}{c}
\dot{\omega}_{s} \\
\mathbf{0} \\
0 \\
S_{V}
\end{array}\right]+\left[\begin{array}{c}
0 \\
\mathbf{J} \times \mathbf{B} \\
\mathbf{J} \cdot \mathbf{E} \\
\mathbf{J} \cdot \mathbf{J} / \sigma
\end{array}\right] .
$$

At the right hand side of Eq. (1), the Lorentz force and the Joule heating terms are included. For the chemical reactions, the eleven species $\left(\mathrm{N}, \mathrm{O}, \mathrm{N}_{2}, \mathrm{O}_{2}, \mathrm{NO}, \mathrm{N}^{+}, \mathrm{O}^{+}, \mathrm{N}_{2}{ }^{+}, \mathrm{O}_{2}{ }^{+}, \mathrm{NO}^{+}\right.$, and $\mathrm{e}^{-}$) are considered through 49 reaction schemes with Park's model. $^{5)}$ For the thermal non-equilibrium, Park's two temperature model $^{5)}$ is employed. The vibrational-translational relaxation process is evaluated with Millikan-White semiempirical model. The viscous and heat transfer coefficients are evaluated with Wilke's mixing rule model. ${ }^{6}$

In the present study, the induced magnetic field is not considered because the magnetic Reynolds number is assumed to be sufficiently small. The electric current density $\mathbf{J}$ is calculated by the following relationship using the generalized Ohm's law considering the Hall effect,

$$
\mathbf{J}=\sigma(\mathbf{E}+\mathbf{V} \times \mathbf{B})-C_{H}(\mathbf{J} \times \mathbf{b}) .
$$

Here, the ion slip effect is not considered for simplicity. The electric current must satisfy the electric current continuity condition under the low magnetic Reynolds number assumption,

$$
\nabla \cdot \mathbf{J}=0 \text {. }
$$

The electric field is evaluated by introducing the electric potential $\phi$, as follows,

$$
\mathbf{E}=-\nabla \phi
$$

By substituting Eqs. (2), (4) into Eq. (3), the Poisson equation for the electric potential ${ }^{7)}$ is obtained and it is solved to evaluate the electric field.

In this study, the interaction of the applied magnetic field and a weakly ionized plasma flow is considered. Here the electric conductivity $\sigma$ and the Hall parameter $C_{H}$ are estimated by the following relationship,

$$
\sigma=\frac{e^{2} N_{e}}{m_{e} v} \quad C_{H}=\frac{e|\mathbf{B}|}{m_{e} v},
$$

where $v$ is the electron collision frequency calculated by

$$
v=\sum_{s \neq e} N_{s} \sigma_{e s}\left(\frac{8 k T_{e}}{\pi m_{e}}\right)^{1 / 2},
$$

\subsection{Numerical methods}

The governing equations described above are discretized by the finite volume method. For the evaluation of the convective term, AUSM-DV scheme ${ }^{8)}$ with 2nd-order spatial accuracy by means of MUSCL approach is employed. The viscous term is evaluated with the central difference method. To resolve the numerical stiffness originated from the chemical reactions, the diagonal point implicit method is employed.9) For the time integration, the Euler explicit method with the local time step is employed.

To solve the Poisson equations for the electric potential, ILU(0)-BiCGstab method ${ }^{10)}$ is employed. The calculation of the Poisson equations is performed 200 iteration cycles after every 1000 steps of the fluid calculation.

\subsection{Body and magnetic field configuration}

In the present study, the MUSES-C capsule with the multiple magnetic source is assumed as shown in Fig. 2. The body geometry is a MUSES-C capsule $(0.4[\mathrm{~m}]$ in diameter, $0.2[\mathrm{~m}]$ in length) which has a spherical head and 45 [deg.] cone in front.

For the applied magnetic field, the dipole magnetic field with $1[\mathrm{~T}]$ in the maximum intensity at the capsule surface is assumed. This intensity is stronger than the one of the single strongest permanent neodymium magnet which has up to 


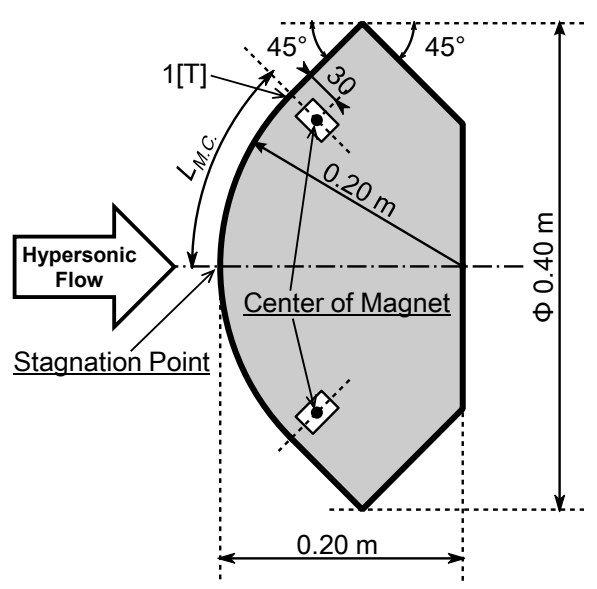

Fig. 2. Computational model.

$0.6[\mathrm{~T}]$, but the Halbach array type of magnetic field source using permanent magnets is considered here to enhance the magnetic field intensity. To obtain the stronger magnetic field, Halbach arrays are practically employed for the high magnetic field generator with permanent magnets, such as Magnetic Resonance Imaging (MRI) scanners and electric motors, which have up to $1[\mathrm{~T}]$ or higher. Actually, many manufactures using the Halbach array exist. ${ }^{11)}$ In the Halbach arrays, the magnetic field is enhanced by design of the appropriate magnetic circuit using several magnets. Therefore, the magnetic field intensity assumed here could be achieved by Halbach array using the present permanent magnet. Of course, the suitable Halbach array should be examined and the specific magnetic field configuration and intensity generated by the magnetic circuit should be estimated, but it is future work. In the present simulation, the dipole magnetic field is assumed for simplicity, which is evaluated by,

$$
\mathbf{B}=\frac{\mu_{0}}{4 \pi}\left[\frac{3 \mathbf{r}(\mathbf{r} \cdot \mathbf{m})-r^{2} \mathbf{m}}{r^{5}}\right],
$$

where, $r$ and $\mathbf{r}$ are measured from the magnet center.

As another parameter for the magnetic field, the magnetic moment which represents the strength of the magnet is considered. The total of the dipole magnetic moment is the same in the single magnet case and all multiple magnet cases. That is, it means the single strong magnet is divided into several weaker magnets in the multiple magnet case. Here, the dipole magnetic moment value determines the distance from the surface to the dipole center. In the multiple magnet case, the dipole magnetic field center is placed equiangularly around the body axis and the dipole moment vector is toward the surface and normal to the surface. The number of magnets is selected to be 8 for the all multiple magnet cases as typical cases. Note that the qualitative trend is not changed even if the number of magnets is varied. For comparison, the single magnet case is also calculated, which has a dipole magnetic field with the same maximum magnetic field intensity at the stagnation point, $1[\mathrm{~T}]$, and this center is $60[\mathrm{~mm}]$ apart from the stagnation point along the body axis. Note that this single magnet condition is similar to the planning flight demonstration experiment capsule using a second stage sounding rocket. ${ }^{12)}$ On the other hand, the dipole center in the
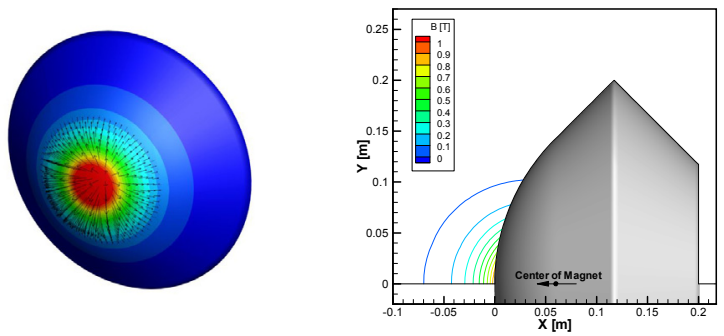

(a) Single magnet case
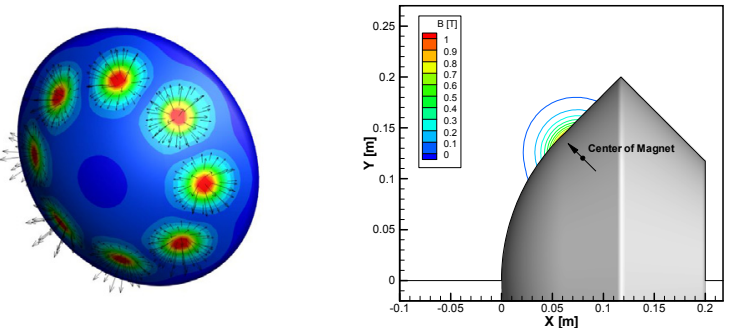

(b) Multiple magnet case $\left(L_{\text {M.C. }}=0.65\right)$
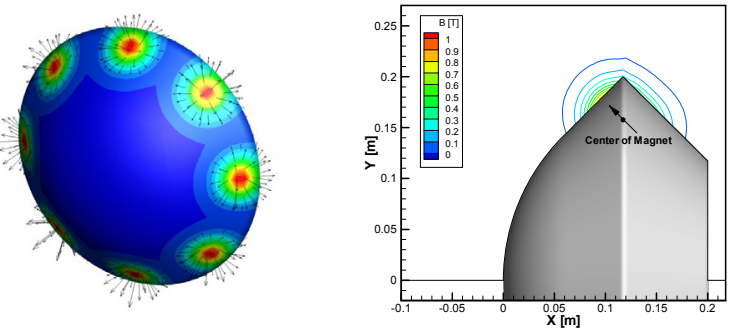

(c) Multiple magnet case $\left(L_{\text {M.C. }}=0.88\right)$

Fig. 3. Applied magnetic field intensity distributions (left; on the capsule surface, right; on the $X-Y$ plane passing through the magnet center).

Table 1. Uniform flow condition.

\begin{tabular}{lcc}
\hline \hline Velocity & 6500 & {$[\mathrm{~m} / \mathrm{s}]$} \\
\hline Angle of Attack & 0 & {$[\mathrm{deg}]$.} \\
\hline Density & $9.48 \times 10^{-5}$ & {$[\mathrm{~kg} / \mathrm{m} 3]$} \\
\hline Static pressure & 6.07 & {$[\mathrm{~Pa}]$} \\
\hline Temperature & 222 & {$[\mathrm{~K}]$} \\
\hline Gas constant (for ideal gas) & 286 & {$[\mathrm{~J} /(\mathrm{kg} \cdot \mathrm{K})]$} \\
\hline Specific heat ratio (for ideal gas) & 1.41 & {$[-]$} \\
\hline \hline
\end{tabular}

multiple magnet cases is $30[\mathrm{~mm}]$ apart from the surface because the total magnetic dipole moment is equal to the one of the single magnet case. That is, each magnet has one-eighth of the dipole magnetic moment of the single magnet case. Figure 3 shows the applied magnetic field distributions in the case of single magnet and multiple magnet cases. The multiple magnet cases are distinguished by the magnet location $L_{M . C}$, which is measured from the stagnation point to the location of the maximum magnetic field intensity along the surface, as shown in Fig. 2, and normalized by the shoulder location. In the single magnet case, the magnetic field is mainly applied near the stagnation point. In contrast, the wider magnetic field appears near the surface in the multiple magnet case. However, the spatial distribution away from the surface is narrower because the magnetic dipole moment is small. In the case of 


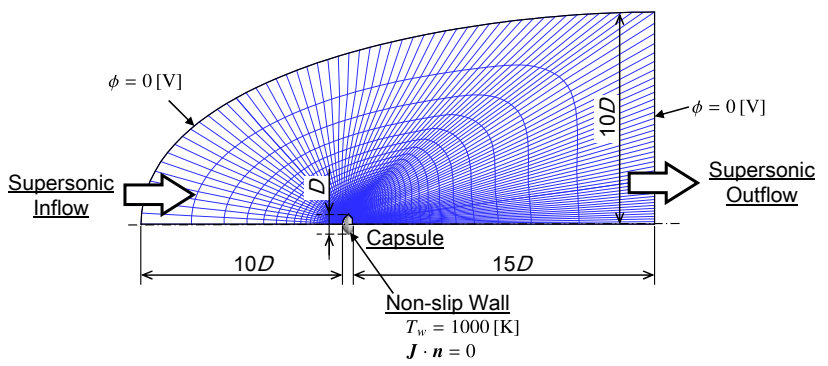

Fig. 4. Computational grid.

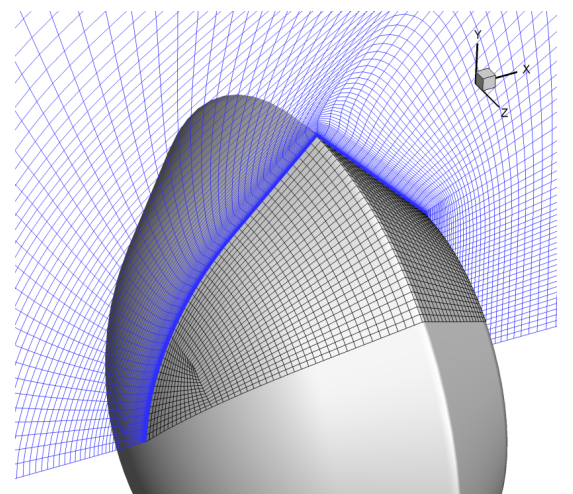

Fig. 5. Computational domain and boundary conditions.

Fig. 3(c), the magnetic field is applied around the shoulder of the capsule, and then, it is also observed in the aft region.

\subsection{Boundary condition and computational grid}

For the flow condition, the Earth's atmospheric entry condition at the altitude of $69[\mathrm{~km}]$ is assumed as shown in Table 1. Figures 4 and 5 show the computational gird and region, respectively. Because of the symmetry of the magnetic field, the calculation is performed on one-eighth part of the entire region, and the circumferential periodic boundaries are used. The computational grid has about 2,600 points on the surface and total about 262,000 points. The wide computational region as shown in Fig. 5 is employed to avoid the influence of the far boundary where the electric potential is set to be $0[\mathrm{~V}]$. For the capsule surface, the non-slip, non-catalytic, and insulation wall $(\mathbf{J} \cdot \mathbf{n}=0)$ conditions are employed. The surface temperature is fixed and set to be $1000[\mathrm{~K}]$.

\section{Results under Real Flight Condition}

Figure 6 shows the influence of the magnetic field configuration on the drag coefficient $C_{D}$ which consists of two parts; the aerodynamic (blue) and electromagnetic (red) drag parts. In this figure, the black dashed line indicates the result of the no magnet case. The total drag coefficient of the all magnet cases is almost equivalent to the one of the no magnet case. In the single magnet case and several multiple magnet cases, the electrodynamic drag part is rather small but it could be observed. In contrast, if the magnet location is away from the stagnation point, the electromagnetic drag part is very tiny. Thus, the clear electrodynamic effect could not be obtained in the present computational condition.

By the way, if the Hall effect is intentionally ignored in the

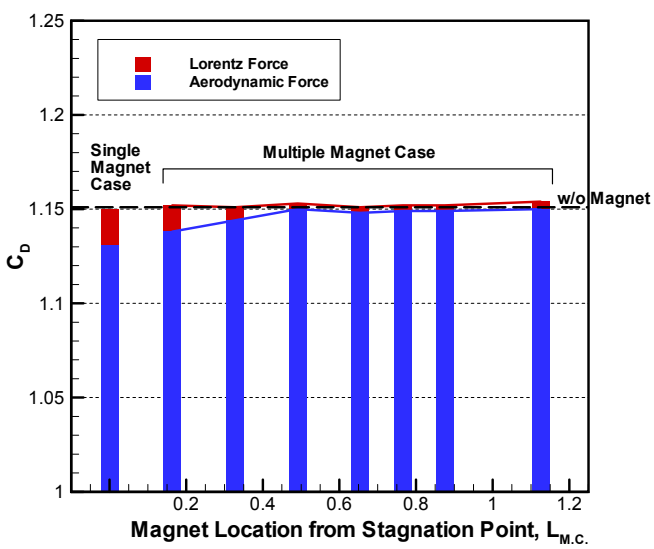

Fig. 6. Influence of the magnetic configuration on the drag coefficient estimated by the MHD simulation considering the Hall effect.

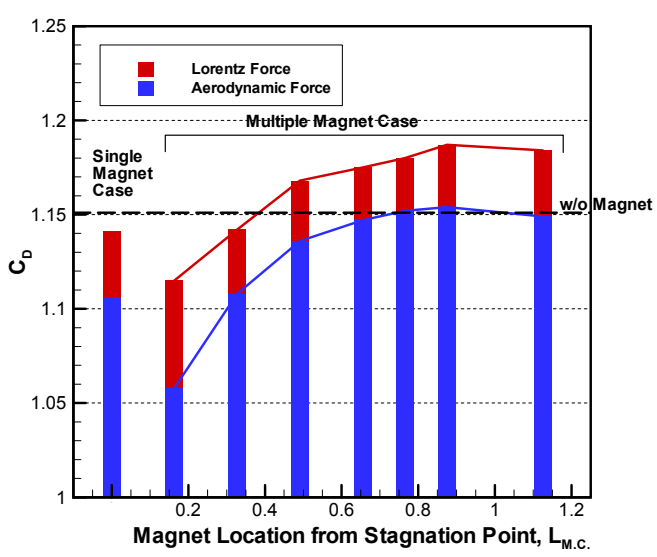

Fig. 7. Influence of the magnetic configuration on the drag coefficient estimated by the MHD simulation not considering the Hall effect.

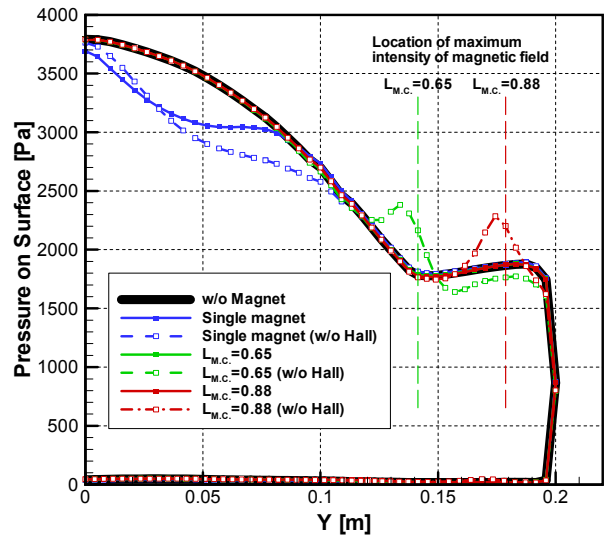

Fig. 8. Comparison of the pressure profile on the surface along the intersection line with the $X$ - $Y$ plane.

calculation, the drag coefficient is clearly affected by the magnetic field configuration, as shown in Fig. 7. This trend is also observed in the pressure distribution on the surface, as shown in Fig. 8. The variation of the pressure distribution could be caused by the electrodynamic effect and it is clearly observed if the Hall effect is ignored. However, considering the Hall effect, this variation is almost disappeared except the single magnet case. Therefore, the Hall effect could 


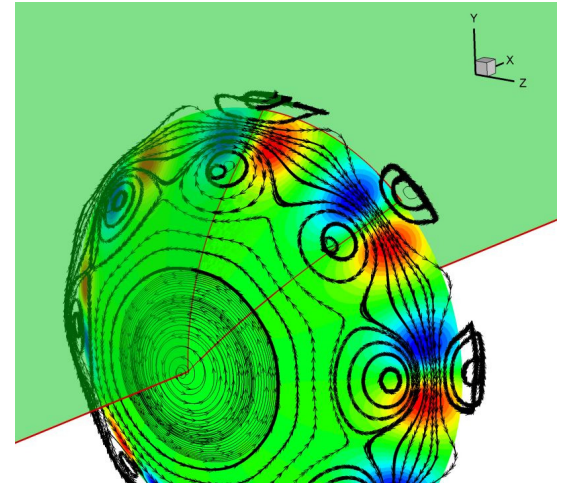

(a) without Hall effect

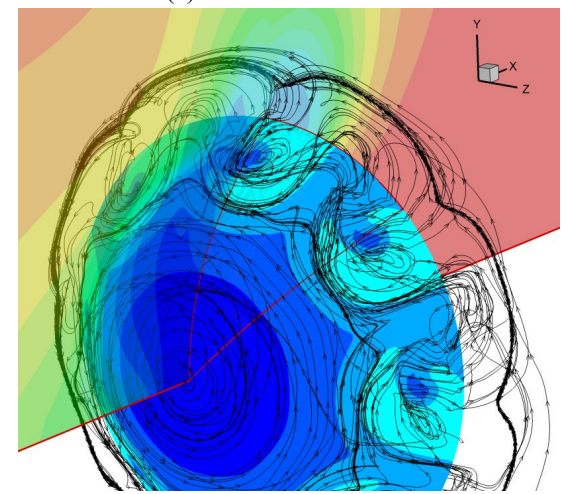

(b) with Hall effect

Fig. 9. Effect of the Hall effect on the induced electric current path and the electric potential contour on the surface in the multiple magnet case $\left(L_{\text {M.C. }}=0.88\right)$

significantly affect the electrodynamic effect caused by the present magnetic configuration. In the single magnet case, the electrodynamic effect is observed for the insulating wall capsule even if the Hall parameter is high, which is consistent with other computational result. ${ }^{7)}$

The electric current is deflected by the Hall effect, and the electric field could be changed. Figure 9 shows the induced electric current path and the electric potential distribution without and with the Hall effect. In this figure, the red curves indicate the computational region boundary and the plot outside this region is copied from this region. The current path, especially near the magnet, is clearly varied by the Hall effect; in the front view, the current path near the magnet is roughly toward clockwise and anti-clockwise direction in the case without and with Hall effect, respectively. The influence on the electric current intensity is shown in Fig. 10, which shows the circumferential electric current distribution on the $X-Y$ plane passing through the magnet center. The strong current region near the magnet is disappeared if the Hall effect is considered. Therefore, the electric current is significantly weakened by the Hall effect, and thus the electrodynamic effect is also diminished.

\section{Influence of Hall Parameter}

In this section, the detailed influence of the Hall effect is investigated by intentionally changing the Hall parameter. Normally, the Hall parameter is uniquely determined by the flow condition, and thus the flow condition should be changed

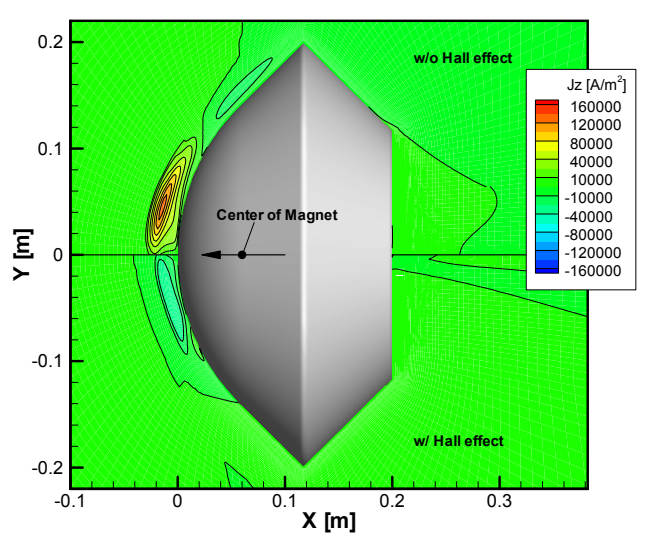

(a) Single magnet case

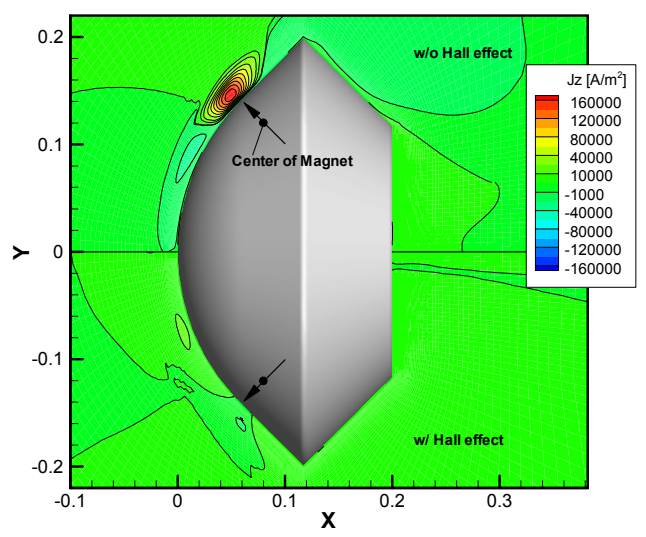

(b) Multiple magnet case $\left(L_{\text {M.C. }}=0.65\right)$

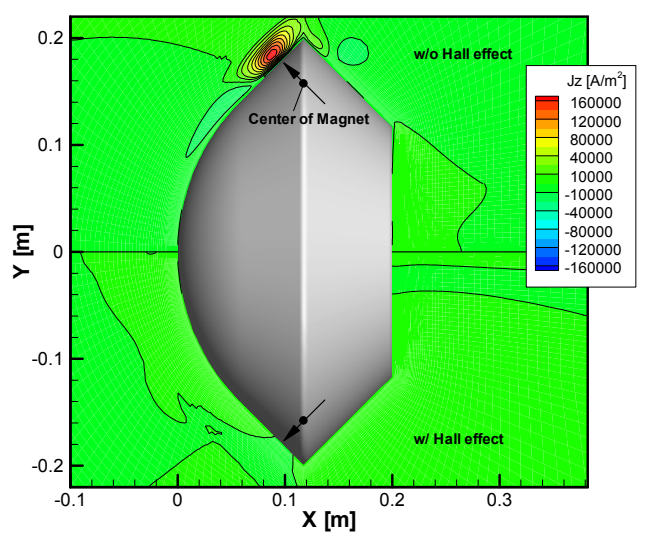

(c) Multiple magnet case $\left(L_{M . C .}=0.88\right)$

Fig. 10. Effect of the Hall effect on the circumferential electric current $J_{z}$ distributions (upper; without, lower; with the Hall effect).

to vary the Hall parameter. However, such results are not clear because it includes the influence of other flow parameters as well as the Hall parameter. Therefore, we carried out another simulation to intentionally vary the Hall parameter, which is based on the ideal gas assumption using the simple model for the ionization gas. As compared with the real gas simulation in the previous section, the gas model is simplified but the Hall parameter can be set arbitrarily and individually. Then, this result gives only qualitative trend of the Hall parameter. The simple model, in which the electric conductivity and the electron number density are dependent only on the 
temperature, is employed as follow, ${ }^{7}$

$$
\sigma=\sigma_{r}\left(\frac{T}{T_{r}}\right)^{2} \quad N_{e}=N_{e, r}\left(\frac{T}{T_{r}}\right)^{2} .
$$

In this study, $\sigma_{r}$ is set to be $200[\mathrm{~S} / \mathrm{m}]$ and $T_{r}$ is set to be $25000[\mathrm{~K}]$, which could be used to approximate the condition within the shock layer under the present flow condition as shown in Table 1. The Hall parameter is rewritten by using this simple model as,

$$
C_{H}=\frac{\sigma_{r} B_{r}}{N_{e, r} e} \frac{|\mathbf{B}|}{B_{r}}=C_{H, r} \frac{|\mathbf{B}|}{B_{r}},
$$

where, $B_{r}$ is set to be the maximum magnetic field intensity within the computational domain, 1[T]. Thus, the Hall parameter is represented by the reference Hall parameter $C_{H, r}$ and the local normalized magnetic field intensity. According to the real gas simulation, $C_{H, r}$ is about 100 near stagnation point for the present flow condition. To investigate the influence of the Hall parameter, $C_{H, r}$ is parametrically varied from 0 to 100 . The body geometry and the magnetic field are the same as the real gas simulation.

Figure 11 shows the influence of the Hall parameter on the total drag coefficient in the single magnet case and multiple magnet cases. In the single magnet case, the drag coefficient is less affected when the Hall parameter is small. On the other hand, the drag coefficient of the multiple magnet cases is significantly affected even if the Hall parameter is small, and it is decreased as the Hall parameter is increased. At $C_{H, r}=50$ or higher, the drag coefficient is almost equivalent to the one of the no magnet case. This result is consistent with the real gas simulation in which $C_{H, r}$ is estimated at 100 . Thus, the electrodynamic effect caused by the present magnetic configuration is significantly affected by the Hall parameter even if the Hall parameter is small.

In the single magnet case, the Lorentz force acting on the flow is rather large because of the strong electric field caused by the Hall effect. ${ }^{7)}$ The Lorentz force is originated by the electric current. The expression of $J_{z}$ is rewritten by the Eq. (2):

$$
J_{z}=\frac{\sigma}{1+C_{H}^{2}}\left\{-C_{H}(\mathbf{E} \times \mathbf{b})_{z}+C_{H} V_{z} \sqrt{B_{x}^{2}+B_{y}^{2}}+(\mathbf{E}+\mathbf{V} \times \mathbf{B})_{z}\right\} .
$$

Here, this equation is satisfied on the $X-Y$ plane where $B_{z}$ and $b_{z}$ is assumed to be 0 because the applied magnetic field is symmetric with respect to the $X-Y$ plane. If the Hall parameter is very large, the first and second terms of the right hand side of Eq. (10) is proportional to $1 / C_{H}$ and the third term is proportional to $1 / C_{H}^{2}$. And, $V_{z}$ of the second term will be relatively small because it represents swirl velocity on the $X-Y$ plane. Thus, the first term including the electric field $\mathbf{E}$ will be important for the electric current when the Hall effect is significant.

The electric potential distribution is affected by the Hall parameter as shown in Fig. 12. These figures show the electric potential distribution on the surface along the intersection line with the $X-Y$ plane in the case of several $C_{H}$. As the Hall

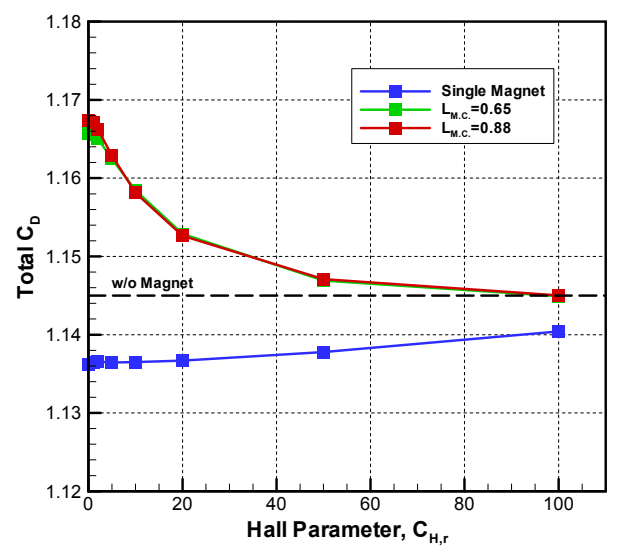

Fig. 11. Influence of the Hall parameter on the total drag coefficient.

parameter is increased, the electric potential becomes low and high at the upstream and downstream of the magnet location, respectively. Thus, the electric field is strengthened as the Hall parameter increasing, which should be correlated with the electric current intensity according to above general behavior. However, the electric field intensity of the multiple magnet case is much weaker than the one of the single magnet case. In the single magnet case, the difference between the maximum and minimum electric potential is increased as the Hall parameter increasing, and then, the electric field is also strengthened. Meanwhile, the one of the multiple magnet case is not increased so much and the electric field is relatively weak. Therefore, the electric current and the Lorentz force are also decreased and the electrodynamic effect will be diminished if the Hall effect is significant.

\section{Conclusion}

In the present study, the influence on the drag force caused by the electrodynamic effect was numerically investigated in the case of the atmospheric entry capsule with the multiple magnetic source which is placed equiangularly around the body axis. As a result of the real gas simulation, the influence on the drag force is very small because the Lorentz force is weakened by the Hall effect if the multiple magnetic fields are applied. To investigate the qualitative influence of the Hall parameter, we carried out another ideal gas simulation in which the Hall parameter can be intentionally varied. As the Hall parameter is increased, the electric current is significantly affected by the Hall effect and the current intensity is weakened because the electric field is generated much weaker than the one of the single magnet case. If the Hall parameter $C_{H}$ is larger than 50 , the drag enhancement effect is almost disappeared for the present computational condition. This result is consistent with the real gas simulation in which $C_{H}$ is estimated at 100. Therefore, when the Hall effect is significant, the present magnetic configuration might not be effective for the drag enhancement.

\section{Acknowledgments}

This research was partially supported by the Japan Society 


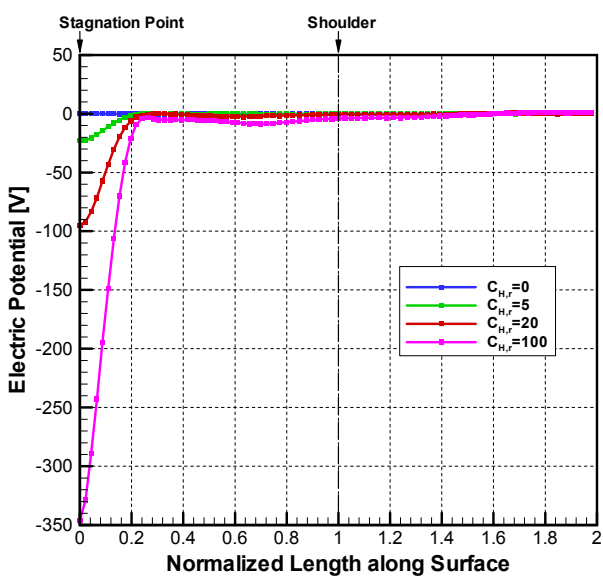

(a) Single magnet case

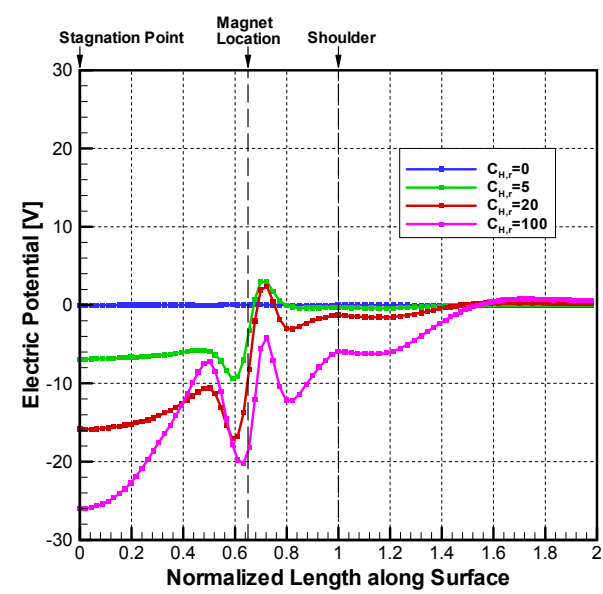

(b) Multiple magnet case $\left(L_{M . C .}=0.65\right)$

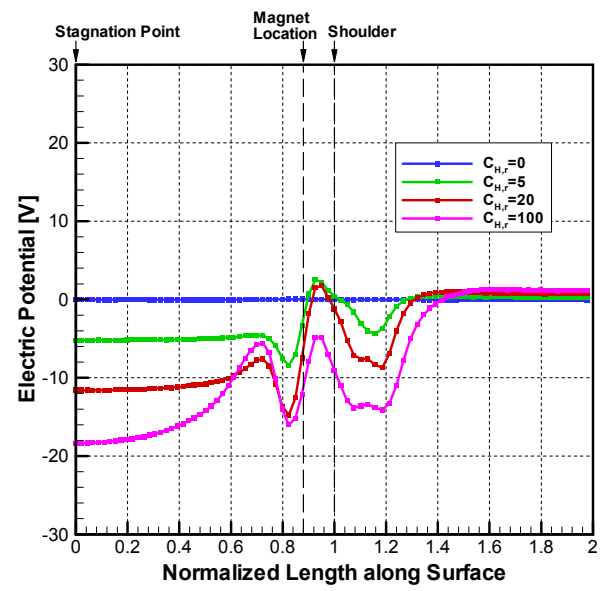

(c) Multiple magnet case $\left(L_{M . C .}=0.88\right)$

Fig. 12. Effect of the Hall parameter on the electric potential profile on the surface along the intersection line with the $X-Y$ plane. for the Promotion of Science, Grant-in-Aid for Young Scientists (B), 26820378.

\section{References}

1) Ziemer, R. W. and Bush, W. B.: Magnetic Field Effects on Bow Shock Stand-off Distance, Phys. Rev. Lett., 1 (1958), pp. 58-59.

2) Bush, W. B.: Magnetohydrodynamic-Hypersonic Flow Past a Blunt Body, Journal of the Aero/Space Sciences, 25 (1958), pp. 685-690.

3) Poggie, J. and Gaitonde, D. V.: Magnetic control of flow past a blunt body: Numerical validation and exploration, Phys. Fluids, $\mathbf{1 4}$ (2002), pp. 1720-1731

4) Katsurayama, H., Abe, T. and Konigorski, D.: DSMC Simulation of Electrodynamic Aerobraking on a Reentry Capsule in a Hypersonic Rarefied Regime, AIAA Paper 2011-3467, 2011.

5) Park, C.: Nonequilibrium Hypersonic Aerothermodynamics, John Wiley and Sons Inc., 1989.

6) Wilke, C. R.: A Viscosity Equation for Gas Mixtures, J. Chem. Phys., 18 (1950), pp. 517-519.

7) Otsu, H., Konigorski, D. and Abe, T.: Influence of Hall Effect on Electrodynamic Heat Shield System for Reentry Vehicles, AIAA J., 48 (2010), pp. 2177-2186.

8) Wada, Y. and Liou, M.-S.: A Flux Splitting Scheme with High-Resolution and Robustness for Discontinuities, AIAA Paper 94-0083, 1994.

9) Eberhardt, S. and Imlay, S.: A Diagonal Implicit Scheme for Computing Flows with Finite-Rate Chemistry, AIAA Paper 90-1577, 1990.

10) van der Vorst, H. A.: Bi-CGSTAB: A Fast and Smoothly Converging Variant of Bi-CG for the Solution of Nonsymmetric Linear Systems, SIAM Journal on Scientific and Statistical Computing, 13 (1992), pp. 631-644.

11) Neomax Engineering Co., Ltd.: Uniform and High Magnetic Field Generator with Permanent Magnet, Hitachi Metals Technical Review, 20, 2004 (in Japaneses).

12) Nagata, Y., Yamada, K. and Abe, T.: Study for Flight Demonstration on Electrodynamic Effect, Symposium on Flight Mechanics and Astrodynamics, Des. 2013 (in Japaneses). 